\title{
Flax roving twisting preparation for weaving and effect of the weft layers number on the physical and mechanical properties of 3D interlock flax fabric
}

\author{
Henri Lansiaux ${ }^{1} \cdot$ Ahmad Rashed Labanieh $^{1}$ (1) $\cdot$ Damien Soulat $^{1} \cdot$ François Boussu $^{1}$
}

Received: 24 May 2019 / Accepted: 15 January 2020 / Published online: 21 January 2020

(c) Springer Nature Switzerland AG 2020

\begin{abstract}
The feasibility to manufacture a 3D warp interlock preform from 1000 Tex flax roving is investigated by means of a prototype machine developed to GEMTEX laboratory. The provided flax roving has low tensile failure load because of lack of twist so it isn't suitable for weaving. The first step was dedicated to improve the tensile properties of the roving through twisting process and identifies the optimal twist level. Experimental campaign is conducted by applying different twist level and evaluates the roving tensile properties. The ultimate tensile load increases as roving twist level increases until a threshold at which the tensile load declines as twist level increases. While the failure strain increases continuously as twist level increases. Five fabrics are produced with the same flax roving, 3D weave architecture and warp number and weft column number per unit length. Only the number of weft layers varies. The physical and tensile properties of these five flax fabrics are characterized experimentally. A considerable effect on the thickness and areal density of the fabric is reported. The difference in the waviness length between the two types of the warp roving (reinforcing and binding) has a significant impact on the tensile behavior of the fabrics in warp direction through the appearance of two phases on the force-strain curve. Otherwise, the variation of the number of layers does not induce a remarkable impact of the fabric structure on the tensile performance of the constituting roving.
\end{abstract}

Keywords Natural fiber $\cdot 3 \mathrm{D}$ weaving $\cdot$ Composite reinforcement

\section{Introduction}

The man-made-fibrous reinforced composite is used widely in different technical application such as aeronautic, automobile and sport goods. They replace the metallic materials thanks to their high specific mechanical properties and high corrosion resistance. However, the man-made fiber does not respond to the growing ecological concern and the material recycling requirement. The natural bast fibers, such as flax, hemp and jute, represent an interesting renewable alternative with less environmental impacts [1]. Further they show better specific stiffness and strength compared to glass fiber [2] and very attractive vibration damping properties [3]. However, these cellulose fibers are sensitive to humidity condition [4] and their chemical compositions limits the temperature at which they can be processed [5]. Moreover, contrary to the the man-made fibers, which have a very long length and are assembled parallel to each other within the roving structure, the bast fibers, which possess specific variable length, are twisted together or sized with adhesion agent to form a continuous roving. The number of twist still a challenge in the roving and preform manufacturing processes because it impacts highly the quality of the manufactured composite regarding the porosity content and lost of the mechanical performance due to the fibers disorientation. Therefore, the development of suitable staple spun roving for the composite reinforcement

Ahmad Rashed Labanieh, ahmad.labanieh@ensait.fr | ${ }^{1}$ ENSAIT, GEMTEX, 59100 Roubaix, France. 
manufacture still a challenging research domain. The bast natural fibers are used currently in form of mat of randomly oriented fibers, unidirectional prepreg, 2D woven fabric and non-crimp fabric [6-8]. However, the potential of use of the natural fiber to manufacture 3D preform is not completely investigated. The 3D preform is composed of different in-plane fibrous layers combined by a through thickness fiber reinforcement [9], which attributes a good delamination resistance and improves the impact damage tolerance of the structure [10]. 3D warp interlock weaving technology is developed to form 3D woven preform based on the conventional weaving process. It consists in combining several layers of orthogonal in-plane rovings using a warp roving passing into the trough-the-thickness direction of the preform and interlaced with the weft roving in a specific weave pattern [9]. The preform is obtained with a fiber reinforcement in through-the thickness direction directly on the weaving machine without need to an additional assembling process further. That is not the case for the laminate manufacturing process where several unidirectional or 2D woven lamina are combined together after overlapping in different in-plane orientations by using stitching or tufting technology [11] which causes fiber damage $[12,13]$. The weavability of flax roving into 3D warp interlock preform is not completely investigated and the adequate spinning and weaving process settings for the composite reinforcement manufacture is not sufficiently explored. In the present study, flax roving is used to manufacture a 3D woven preform. The effect of the twist level of dry roving on its mechanical performance is evaluated in order to find the optimal value responding to the weaving process requirements. After twisting the roving with a selected twist level, five structures of different number of the weft layers are woven with keeping constant all other weaving parameters. The effect of the number of layers is examined on the geometrical and physical properties, in addition to the behavior of the dry fabric under uni-axial quasi-static tensile load, are explored.

\section{Material and method}

\subsection{Roving}

The used flax roving for the preform fabrication are provided by 'Depestele' company with a linear density of $1057 \pm 41.4 \mathrm{Tex}(\mathrm{g} / \mathrm{Km})$ verified according to NF G07-316. No twist is inserted to the roving but an adhesion agent is applied to ensure the roving cohesion for handling. The provided roving has not the sufficient tenacity to sustain the applied loads during the weaving process. On the weaving loom, warp undergoes dynamic tensile loads generated by the shed formation operation and inter-roving friction and roving/reed friction caused by the packing operation. Therefore additional twist has to be inserted to the roving before weaving in order to improve its tenacity. The optimal twist level is that which is sufficient to respond to the weaving requirements without degrading the quality of the composite regarding the potential permeability of the roving for resin due to roving compaction and the expected stiffness and strength due to the fiber disorientation. For this reason, an evaluation of the effect of the twist level on the mechanical performance of the roving has been conducted. The following twist level values have been inserted: $30,45,60,75,90$ and 100 tpm using 'Twistec TW 4/300' twisting machine. After twisting, the real twist level on the roving is verified according to NF ISO 2061. Then uni-axial quasi-static tensile test is performed according to NF ISO 2062.

\subsection{Woven fabric}

The roving, with the selected twist level, is used to produce 3D warp interlock woven fabric by means of a special weaving loom built in GEMTEX Laboratory [14]. A specific definition method has been proposed by Boussu et al. [9] to describe the architecture of 3D warp interlock woven fabric and the position of the constituting roving inside the fabric. This definition method is respected in the present work when defining the produced fabric and assigning the identification label. The definition is comprised of a series of letters and numbers defined as the following:

$X_{1} X_{2} N Y_{1}-Y_{2}\{$ Binding $\}$

- $X_{1}:$ Type of angle of binding warp yarn, $\mathrm{O}$ (orthogonal) or A (angle)

- $X_{2}$ : Type of depth of the binding warp yarn, L (layer to layer) or $\mathrm{T}$ (through the thickness)

- $\quad N$ : Number of weft layers for each column of the 3D warp interlock fabric elementary pattern

- $Y_{1}$ : Binding step, corresponds to the number of wefts between to interlacing points of binding yarn in the same layer

- $Y_{2}$ : Binding depth, corresponds to the number of weft yarns layers linked with the binding warp yarn

- $\{$ Binding\}: Weave pattern of binding warp yarns

Five structures are manufactured with variation only the number of weft layers $(N=3,5,7,11,23)$. The choice of these values of the layer number is related to the number of heald shafts (24) on the loom and the weave architecture condition of the elementary unit consisting of having one reinforcing warp between two weft layers. For instance, with 7 weft layers the elementary weave unit of 
the fabric contains 6 layers of reinforcing warp and two binding warp yarns. So this unit is repeated 3 times on the 24 shafts of the loom. All these structures are produced with considering orthogonal angle $\left(X_{1}=0\right)$ and through-the-thickness depth path $\left(X_{2}=T\right)$ for binding that is interlaced with wefts according to the plain weave pattern. Since the binding depth pattern is through-thethickness thus the value of the binding depth $\left(Y_{2}\right)$ is equal to the number of weft layers $(N)$. While the binding step $\left(Y_{1}\right)$ is equal to 1 for all structures. Figure 1 shows the weave curve of O-T 3 1-3 \{plain weave\} fabric and a sample of the fabric on the weaving loom. The warp is drafted on the heald shafts of the loom with 6 rovings per centimeter. After weaving the number of rovings per unit length is counted according to ISO 4602 Standard in both warp and weft fabric directions. Further, the roving crimp inside the five structures is measured for the three constituting rovings (reinforcing warp binding warp and weft) according to ISO 711-3 standard. The thickness, area density and tensile behavior in the two main fabric directions are evaluated for the five structures according to ISO 4603, ISO 12127 and ISO 13934-1 standards respectively.

\section{Results and discussion}

\subsection{Effect of twist on the tensile properties of the roving}

Twisting brings the fiber in helix path inside the yarn structure and the angle of the helix relative to the yarn main axis is directly proportional to the twist level. In general, extending a twisted yarn exhibits three types of deformation mechanism which can be pointed out on the force-strain curve. The beginning of the curve is characterized by a zone of low resistance associated with high strain. It corresponds to redressing the fibers inside the yarn into the load direction resulting in extend the fiber helix and decrease the twist angle. That leads to increase the lateral compaction between the fibers and the inter-fiber friction force which causes the fiber deformation and generates a reaction force by the fibers. This reaction force depends on the fiber stiffness and the induced strain. At this stage, without exceeding the fiber strength, the inter-fiber sliding occurs when the reaction force overcomes the inter-fiber static friction force threshold. Figure 2 presents the force-strain curve of flax roving obtained from testing the twisted flax roving at different twist level. To illustrate the effect of twist, the force and strain at break are plotted as a function of the real twist level (measured after twisting) on Figs. 3 and 4 respectively. The increase of twist angle causes a direct increase in the breaking force because of the increase of the lateral compaction between the fibers and the inter-fibers friction forces with the twist angle [15]. However, a threshold is observed at a twist level around 80 tpm where beyond this level the breaking force stagnates even with the increase of twist level. At this stage, it is supposed that the fiber reach the maximum level of redressing but they still highly disoriented relative

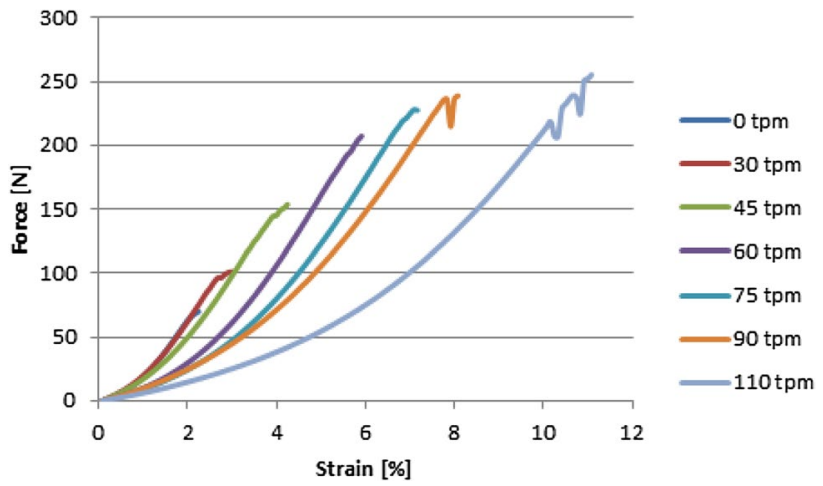

Fig. 2 Force versus strain curve of the tested flax rovings at different twist levels

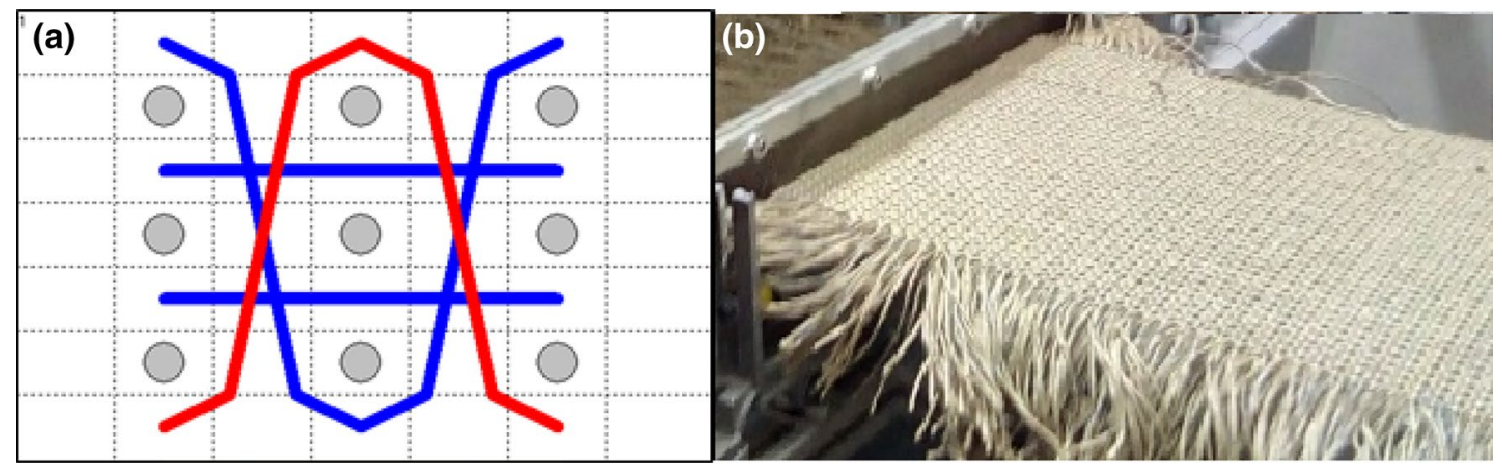

Fig. 1 Cross section scheme view of 3D warp interlock O-T 3 1-3 \{plain weave\} (a), produced fabric on the weaving loom built in GEMTEX Laboratory (b) 


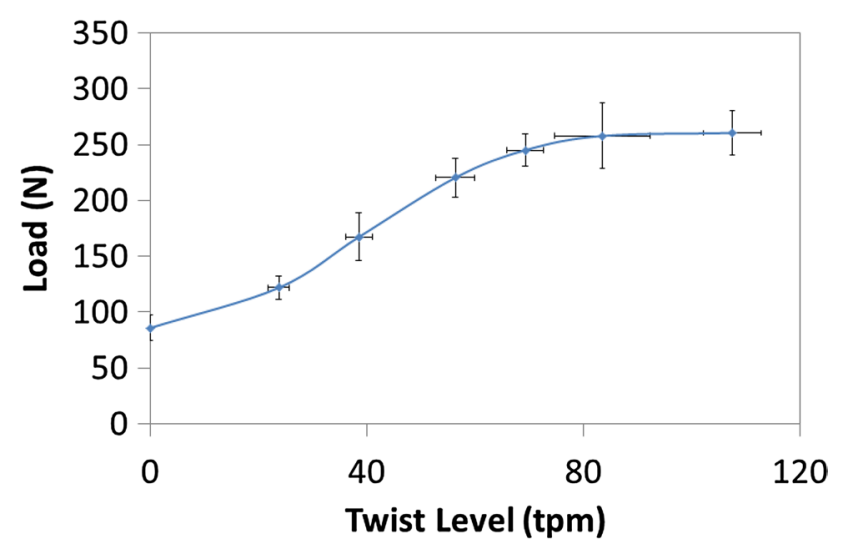

Fig. 3 Breaking force versus twist level of the used flax roving

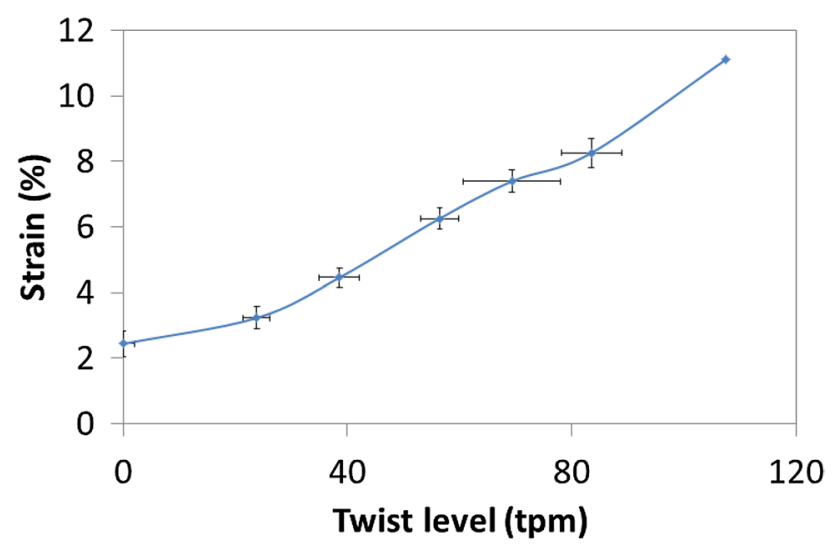

Fig. 4 Breaking strain versus twist level of the used flax roving curve, as observed on Fig. 2. In another part, it is supposed that the inter-fiber sliding mechanism takes more place in the yarn behavior and the fiber deformation is accompanied with low tensile force due to the high fiber disorientation.

\subsection{Effect of number of layers on the physical properties of fabric}

All structures are produced on the same weaving loom without changing the drafting system and with the same number of warp per centimeter across the fabric width (6 rovings/cm). Since the binding step is equal to 1 roving and the path of the binding warp roving is orthogonal with through-the-thickness depth type following a plain weave pattern so the fabric elementary weave unit is composed of two columns of weft. The weaving machine is set to obtain 2 columns of weft roving per centimeter for all the structures. Thus, for the given architecture characteristics and weaving parameters, the number of weft roving per centimeter is expected to increase by 2 rovings per layer. However, this factor is not exactly respected on the produced fabrics due to weaving process restrictions and compaction of the roving and the fabric, Table 1 . The variation of the number of weft per centimeter as a function of the number of layers with the same number of the warp roving per centimeter conduces to change the ratio of the fiber content between the fabric main directions (warp and weft), Table 2. Otherwise, the distribution of the warp
Table 1 Physical properties of the manufactured 3D interlock woven fabrics as a function of the number of layers

\begin{tabular}{llcl}
\hline Structure & Number of weft $/ \mathrm{cm}$ & Thickness $(\mathrm{mm})$ & Areal density $\left(\mathrm{kg} / \mathrm{m}^{2}\right)$ \\
\hline O-T 3 1-3 & $5.18(0.19)$ & $5.11(0.26)$ & $1.25(0.03)$ \\
O-T 5 1-5 & $8.10(0.20)$ & $5.37(0.27)$ & $1.58(0.02)$ \\
O-T 7 1-7 & $12.21(0.22)$ & $6.81(0.40)$ & $2.04(0.02)$ \\
O-T 11 1-11 & 25.00 & $10.03(0.35)$ & $3.49(0.04)$ \\
O-T 23 1-23 & 54.60 & $18.00(1.00)$ & $6.34(0.12)$ \\
\hline
\end{tabular}

(SD) The standard deviation of the measured values is writen into brackets the applied charge. And since the roving reaction force acquired on tensile machine is equivalent to the component of the generated fiber stress and the inter-fiber friction force in the load direction which is related to the cosine of the twist angle. Thus the increase of twist angle leads to decreas the magnitude of contribution of the internal force. Contrary to the breaking force, the breaking strain doesn't stop increasing with the increase of the twist level Fig. 4 and no threshold is observed. That is attributed in a part to the increase of twist angle leading to extend the first redressing zone on the force-strain
Table 2 Distribution of roving between two fabric directions (warp and weft) and distribution of warp roving between reinforcing and binding type

\begin{tabular}{lll}
\hline Structure & Weft/warp & $\begin{array}{l}\text { Reinforcing } \\
\text { warp/binding } \\
\text { warp }\end{array}$ \\
\hline O-T 3 1-3 & 0.86 & 2 \\
O-T 5 1-5 & 1.31 & 3 \\
O-T 7 1-7 & 1.99 & 4 \\
O-T 11 1-11 & 4.16 & 6 \\
O-T 23 1-23 & 8.96 & 12 \\
\hline
\end{tabular}


Table 3 Crimp percentage of the constituting roving in the different manufactured 3D interlock woven fabric

\begin{tabular}{llll}
\hline Structure & Warp reinforcing & Warp binding & Weft \\
\hline O-T 3 1-3 & $2.1(0.78)$ & $22.3(4.10)$ & $2.7(0.71)$ \\
O-T 5 1-5 & $1.4(0.80)$ & $31.7(6.90)$ & $1.0(1.20)$ \\
O-T 7 1-7 & $0.5(0.51)$ & $48.3(10.05)$ & $1.3(1.06)$ \\
O-T 11 1-11 & $2.2(1.40)$ & $138.8(25.90)$ & $3.3(0.80)$ \\
O-T 23 1-23 & $2.2(0.50)$ & $237.2(12.51)$ & $2.3(1.20)$ \\
\hline
\end{tabular}

(SD) The standard deviation of the measured values is writen into brackets

roving between the two types reinforcing and binding varies directly with the variation of the number of layers in the fabric. The ratio between the number of two warp roving types in the elementary weaving unit is presented in Table 2. The increase of the number of layers conduces also to increase the thickness and areal density of the fabric, Table 1. crimp percentage of the different constituting rovings (warp reinforcing, warp bending and weft) of the five fabricated 3D woven fabric is presented in Table 3. The consumed length of the warp binding roving increases considerably with the number of layers as a result of increasing the fabric thickness. Otherwise, the variation of the number of layers has no significant impact on the crimp of the reinforcing warp and weft rovings.

\subsection{Effect of number of layers on the tensile properties of fabric}

The behaviour of the manufactured 3D warp interlock fabric at dry stat under unidirectional tensile loads is conducted in both main directions of the fabric (warp and weft). In general when extending a dry fabric under a tensile load, the solicited yarns in the load direction exhibits two type of deformation; yarn redressing (fabric structural deformation), dominates the first zone of the force-strain curve, and yarn reaction to the load (yarn deformation), dominates once the yarns redressing reached at the maximum level. Extend of the redressing phase is related to the waviness length of the yarn inside the fabric.

\subsubsection{Tensile behavior in warp direction}

Figure 5 presents force-strain curve of two structures O-T 3 1-3 and O-T 7 1-7 (3 and 7 layers respectively). These structures are selected as an example to illustrate the effect of the number of layers on the fabric tensile behaviour in warp direction. Two phases are observed on both curves referred to the difference in the waviness length between the two group of warp roving (reinforcing and binding

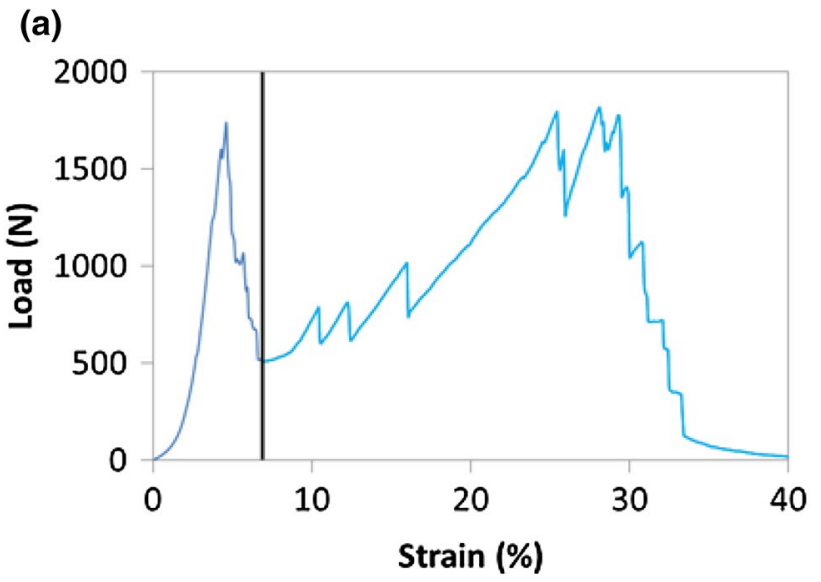

(b)

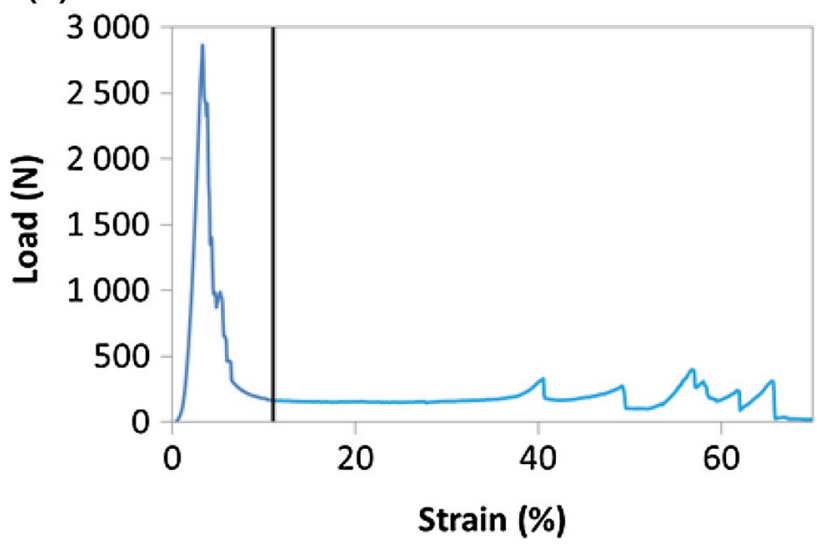

Fig. 5 Force-strain curve of tensile test of O-T 3 1-3 a and O-T 7 1-7 b in warp direction

warp roving). The difference in waviness length inside the fabric structure is shown through the difference of the crimp percentage, Table 3, higher crimp percentage indicates longer waviness length. The two phases on the force-strain curves are highlighted by two different colors on Fig. 5. A distinct peak with high force and low strain, relative to the total fabric strain at break, appears in the first phase. It corresponds to the break of the reinforcing roving. Then the force moves up again with high strain value in the second phase with a series of peaks, which corresponds to the break of the binding roving. The reinforcing warp rovings have quasi-identical length inside the structure, which is deduced by the low standard deviation value of the crimp percentage in Table 3, thus they break at the same time approximately with one peak on the tensile test curve in the first phase. While the length of the binding rovings varies more inside the structure, which is deduced by the higher standard deviation value of the crimp percentage in Table 3, leads to obtain a series of peaks on the tensile test curve. The maximum force value, corresponding to the first peak on the first phase, is more 
important in the fabric possessing higher number of layers. That is explained by the higher ratio of reinforcing warp roving relative to binding one with the same number of warp roving per centimeter for all structures, Table 2 . On the contrary the peaks in the second phase has higher force value in the fabric which possess less number of layers since the number of binding roving is higher. Otherwise the fabric of higher number of layers the peaks in the second phase appears late at high strain value due to the longer waviness length of binding roving associated with higher fabric thickness and crimp percentage, Table 1.

\subsubsection{Tensile behavior in weft direction}

The phenomena of two distinct phases is not observed on the force-strain curve of the tensile test of the manufactured fabrics in the weft direction, Fig. 6. That is attributed to the identical waviness length between the weft rovings, which have the same path inside the fabric structure, Table 3. Otherwise, the redressing zone of weft roving due to the waviness inside the structure, characterized by low force and high strain, is obvious on the force-strain
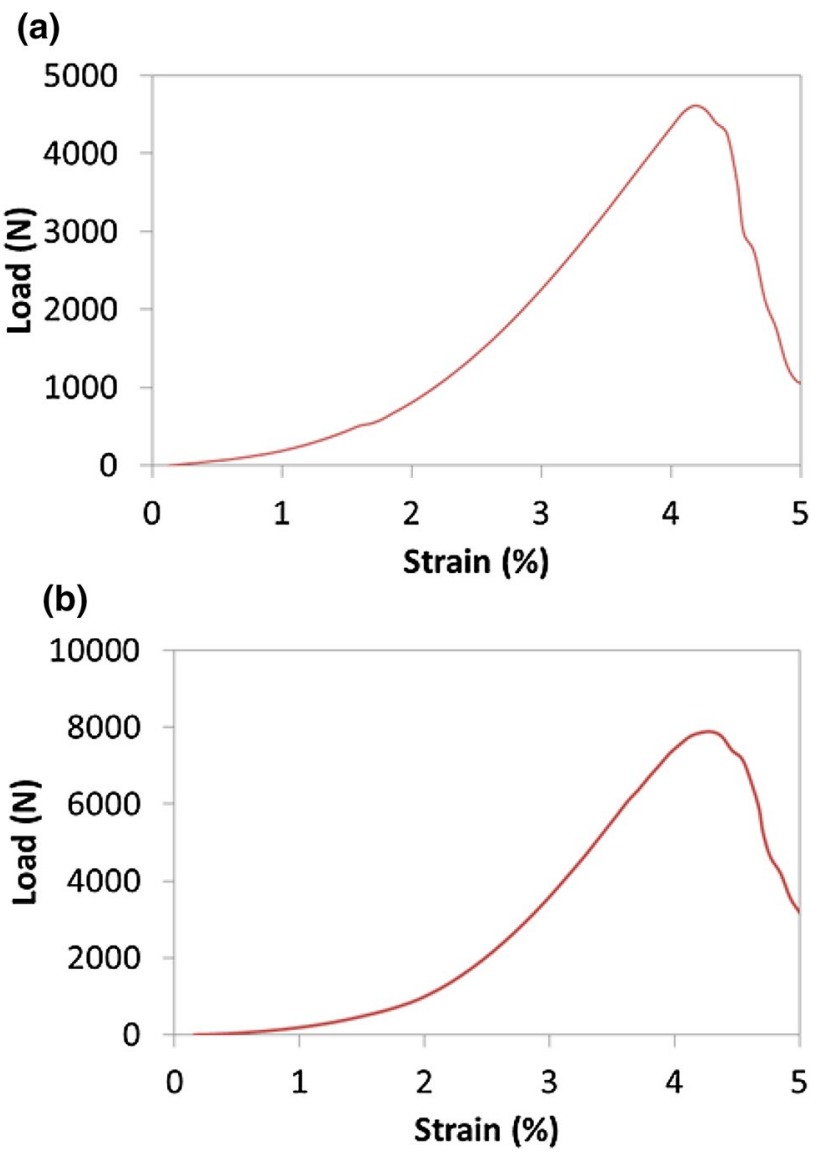

Fig. 6 Force-strain curve of tensile test of O-T 3 1-3 a and O-T 7 1-7 b in weft direction
Table 4 Maximum force and strain at break of the manufactured 3D interlock woven fabric as a function of number of layers

\begin{tabular}{lcl}
\hline Structure & Maximum force $(\mathrm{N})$ & Strain at break \\
\hline O-T 3 1-3 & $4616(639)$ & $4.22(0.17)$ \\
O-T 5 1-5 & $5750(410)$ & $3.94(0.26)$ \\
O-T 7 1-7 & $8114(644)$ & $4.27(0.05)$ \\
O-T 11 1-11 & $17722(1208)$ & $5.33(0.31)$ \\
O-T 23 1-23 & $29356(1514)$ & $9.14(0.78)$ \\
\hline
\end{tabular}

(SD) The standard deviation of the measured values is writen into brackets

curve. Then the roving resistance against the extension load takes place in the second zone, characterized by high increase in force. The maximum tensile force and the strain at break of the fabrics in weft direction as a function of number of layer is presented in Table 4. The maximum forces increases with the number of layers as a result of the increase in the number of weft roving per centimeter for the considered 3D warp interlock architecture, Table 1. However, the rovings across the fabric width don't break simultaneously due to the slight variation of crimp percentage and the scatter in rovings tenacity. Therefore, the force-strain curve does not exhibit a drastic drop in the force value once the maximum force is attained. Thus comparison of the maximum force between the different fabrics is not sufficient to identify the effect of the fabric structure generated by the variation of the number of layer on the tensile performance of 3D warp interlock fabric in weft direction. Therefore, the ratio of the breaking energy of the fabrics, brought back to the number of roving, to the breaking energy of one roving, obtained from the tensile test of a single roving, is reported in Fig. 7. The consideration of this ratio eliminates the effect of number of roving on the executed work for the fabric break and shows the effect of the structure on the tensile behavior of the

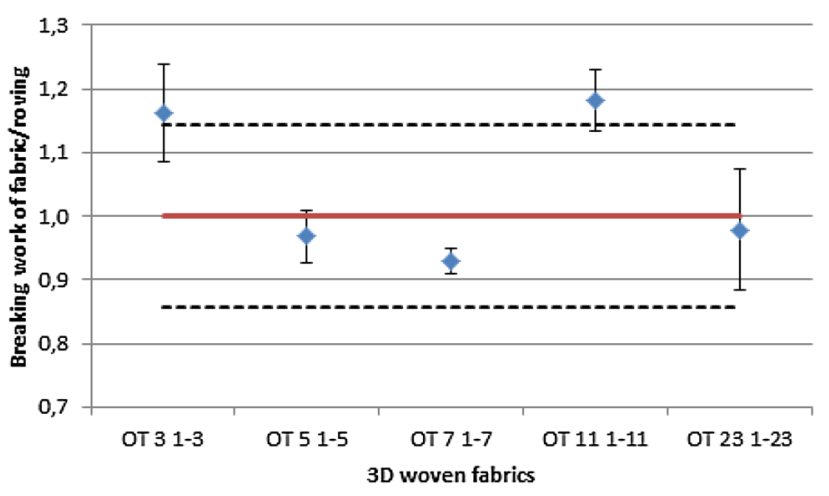

Fig. 7 Ratio between the breaking work of fabric in weft direction referred to the number of roving across the sample and the breaking work of the roving 
roving and the fabric. On Fig. 7 the scatter in the breaking energy of the single used roving is illustrated via the two dashed lines. A ratio equal to one signifies that the breaking energy of the fabric returned to one roving is equal to the breaking energy of a single roving. The obtained value for the different fabricated structures shows that there is no significant effect of the fabric structure related to the variation the number of layers on the roving tensile behavior inside the fabric and on the fabric behaviour in the weft direction.

\section{Conclusion}

Manufacture of 3D warp interlock fabric with a flax roving is explored in this work using a special weaving loom developed in GEMTEX laboratory. The number of layers is varied for the five structures with maintaining all other weaving and structure parameters constants. The impact of the variation of the number of layers on the physical and mechanical properties of the 3D woven fabric is examined. This variation with maintaining the weave pattern and the number of warp roving constant causes structural modifications relative the ratio between the warp and weft rovings within the fabric and the distribution of the warp roving between the reinforcing and binding type. Further, the increase of the number of layers conduces to increases the fabric thickness and its areal density. The force-stain curve issue of the tensile test of the manufactured 3D woven fabric in the warp direction is characterized with two phases. These phases are related to the two warp yarn groups and their crimp length inside the fabric structure. The two phases phenomena does not show up when extending the fabric in the weft direction. The breaking force of the fabric depends on the number of the weft roving which increases with the number of layers. However, no significant effect of the fabric structure is observed on the roving and fabric behavior when comparing the executed work to break the fabric in weft direction brought back to the number of roving relative to the work done to break one single roving. Twisting of the roving has a considerable effect on its mechanical behavior during weaving process and the properties of the composite. Therefore, an experimental study has been conducted to evaluate the effect of the twist level on the tensile behavior of the used dry roving. The breaking force of the roving under tensile load increases with the twist level until a critical value where the force stagnates.

Acknowledgements This project has received funding from Hautsde-France region, French Environment and Energy Management Agency (ADEME) and Depestele group providing the used flax roving.

\section{Compliance with ethical standards}

Conflict of interest The authors declare that they have no conflict of interest.

\section{References}

1. Pickering KL, Efendy MGA, Le TM (2016) A review of recent developments in natural fibre composites and their mechanical performance. Compos Part A Appl Sci Manuf 83:98-112

2. Pil L, Bensadoun F, Pariset J, Verpoest I (2016) Why are designers fascinated by flax and hemp fibre composites. Compos Part A Appl Sci Manuf 83:193-205

3. Fabien D, Pierre-Etienne B, Plummer CJG, Månson J-A (2014) Damping of thermoset and thermoplastic flax fibre composites. Compos Part A Appl Sci Manuf 64:115-23

4. Reux F, Verpoest I (2012) Flax and hemp fibres: a natural solution for the composite industry. JEC Composites and CELC, Press Release at JEC Europe-Composites Show \& Conférences, Paris, 27-29 March

5. Vertommen J (2014) Manufacturing of chemically treated thermoplastic flax fibre composites. Master thesis KU Leuven, Faculty of Engineering Technology, Campus Group T

6. Cuynet A, Scida D, Roux E, Toussaint F, Ayad R, Lagache M (2018) Damage characterisation of flax fibre fabric reinforced epoxy composites during low velocity impacts using high-speed imaging and stereo image correlation. Compos Struct 202:1186-1194

7. Capelle E, Ouagne P, Soulat D, Duriatti D (2014) Complex shape forming of flax woven fabrics: design of specific blank-holder shapes to prevent defects. Compos Part B Eng 62:29-36

8. Omrani F, Wang P, Soulat D, Ferreira M, Ouagne P (2017) Analysis of the deformability of flax-fibre nonwoven fabrics during manufacturing. Compos Part B Eng 116:471-485

9. Boussu F, Cristian I, Nauman S (2015) General definition of 3D warp interlock fabric architecture. Compos Part B Eng $81: 171-188$

10. Mouritz AP, Cox BN (2010) A mechanistic interpretation of the comparative inplane mechanical properties of 3D woven, stitched and pinned composites. Compos Part A Appl Sci Manuf 41:709-728

11. Tong L, Mouritz AP, Bannister MK (2002) 3D Fibre reinforced polymer composites. Elsevier Science Ltd, Amsterdam

12. Hes $\mathrm{H}$ et al (2007) Elastic constants estimation of stitched NCF CFRP laminates based on a finite element unit-cell model. Compos Sci Technol 67(6):1081-1095

13. Mouritz AP, Cox BN (2000) A mechanistic approach to the properties of stitched laminates. Compos Part A Appl Sci Manuf 31(1):1-27

14. Boussu F, Veyet F, Dumont N (2009) Développement d'une machine prototype de tissage pour la réalisation d'échantillons textile 2,5D, Private project A0807017N, OSEO

15. Morton WE, Hearle JWS (2008) Physical properties of textile fibres, vol 765, 4th edn. Woodhead Publishing Limited in association with The Textile Institute, Cambridge

Publisher's Note Springer Nature remains neutral with regard to jurisdictional claims in published maps and institutional affiliations. 